\title{
Monte-Carlo Based Sensitivity Analysis of Acoustic Sorting Methods
}

\author{
Gergely Simon', Gergely B. Hantos', Marco A. B. Andrade², Marc P. Y. Desmulliez¹, \\ Mathis O. Riehle ${ }^{3}$, Anne L. Bernassau ${ }^{1 *}$ \\ ${ }^{1}$ School of Engineering and Physical Sciences, Institute of Signals, Sensors and Systems, Heriot-Watt University, \\ Earl Mountbatten Building First Gait, Edinburgh EH14 4AS, United Kingdom \\ 2 Institute of Physics, University of São Paulo, São Paulo, Rua do Matão 1371, Brazil \\ ${ }^{3}$ Institute of Molecular Cell and Systems Biology, Centre for Cell Engineering, University of Glasgow, \\ Joseph Black Building, University Place, Glasgow, G12 8SU, United Kingdom \\ * Corresponding author, e-mail: A.Bernassau@hw.ac.uk
}

Received: 14 November 2018, Accepted: 14 January 2019, Published online: 02 April 2019

\begin{abstract}
Separation in microfluidic devices is a crucial enabling step for many industrial, biomedical, clinical or chemical applications. Acoustic methods offer contactless, biocompatible, scalable sorting with high degree of reconfigurability and are therefore favored techniques. The literature reports on various techniques to achieve particle separation, but these do not investigate the sensitivity of these methods or are difficult to compare due to the lack of figures of merit. In this paper, we present analytical and numerical sensitivity analysis of the time-of-flight and a phase-modulated sorting scheme against various extrinsic and intrinsic properties. The results reveal great robustness of the phase-modulated sorting method against variations of the flow rate or acoustic energy density, while the time-offlight method shows lower efficiency drop against size and density variations. The results presented in this paper provide a better understanding of the two sorting methods and offer advice on the selection of the right technique for a given sorting application.
\end{abstract}

Keywords

sensitivity analysis, Monte-Carlo methods, acoustic sorting, lab-on-a-chip devices

\section{Introduction}

Sorting methods in microfluidic devices are critical enabling technologies for applications in cell biology, biomedicine or industry [1, 2]. While passive methods can be developed for low cost, disposable devices using simple manufacturing steps [3], active methods are preferred for their reconfigurability and adaptation to various target particle or cell populations [4]. Acoustic active methods are favored for their non-contact, biocompatible and label-free properties $[5,6]$. Various solutions exist in the literature for continuous flow sorting, including bulk [7] and surface acoustic wave devices [8], using either travelling [9] or standing waves [10], modulated signals [11] or unique geometries [12].

Although most of the works include analysis of the degree of sorting depending on some of the device parameters such as input power [13, 14], flow rate [14] or particle size [15], none of these attempt to assess the sensitivity of the separation technique with respect to input or operational parameters. Moreover, comparison of the different methods is difficult due to the broad range of frequencies, particle sizes or device dimensions and flow rates used.

To the best of the authors' knowledge, there is only one publication assessing the performance of an acoustic sorting method for several intrinsic (particle dependent) or extrinsic (experimental setup dependent) parameters [16]. However, in this work, only a single sorting method is analyzed, and the device performance is not quantified through the usual figures of merit such as sorting purity or efficiency, but through the possible output position range of the particles. Therefore, our paper carries out a comprehensive sensitivity analysis for two different acoustic sorting methods: the commonly used time-of-flight sorter [14] and a phase-modulated sorting technique [15]. This article also quantifies the device performance using efficiency and purity as figures of merit. 


\section{Acoustic sorting methods}

Particles subjected to acoustic fields in a liquid medium experience acoustic radiation forces due to time averaged second order effects. The viscous drag force of the liquid balances the acoustic radiation force and, as a result of different scaling of the acoustic and hydrodynamic forces with particle size, density or compressibility, different particles follow different trajectories and separation is achieved. With the time-of-flight approach, the particles are exposed to acoustic standing waves that are perpendicular to the flow direction, and different particles move laterally by different magnitudes allowing them to be extracted at different outlets. In the phase-modulated method, a peculiar phase pattern is used to move the standing wave pattern, resulting in separation of the particles.

\subsection{Time-of-flight methods}

Taking the reference frame as shown in Fig. 1, i.e. the $x$ axis along the device length and $y$ axis along the cross-section, the acoustic radiation force in a standing wave can be expressed as:

$$
\begin{aligned}
& F_{\text {ac }, y}=c_{\mathrm{ac}} \sin \left(2 k_{y} y\right) \\
& \text { with } \\
& c_{\mathrm{ac}}=E_{\mathrm{ac}} V_{\mathrm{p}} \Phi_{\mathrm{AC}} k_{y} \\
& \Phi_{\mathrm{AC}}=\left[f_{1}+\frac{3}{2} \operatorname{Re}\left[f_{2}\right] \frac{k_{y}^{2}-k_{z}^{2}}{k^{2}}\right] \\
& f_{1}=1-\kappa_{\mathrm{p}} / \kappa_{0} \\
& f_{2}=\frac{2\left(\rho_{\mathrm{p}} / \rho_{0}-1\right)}{2 \rho_{\mathrm{p}} / \rho_{0}+1}
\end{aligned}
$$

where $E_{\mathrm{ac}}$ is the acoustic energy density, $V_{\mathrm{p}}$ the particle volume, $\Phi_{\mathrm{AC}}$ the acoustic contrast factor, and $k_{i}$ the wavenumbers [17]. The constants $f_{1}$ and $f_{2}$ are called the monopole

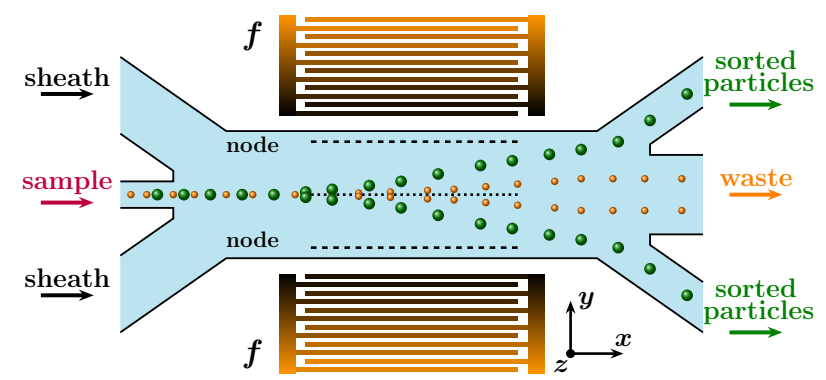

Fig. 1 Illustration of the time-of-flight method. Sample mixture enters aligned with the antinode (dotted line) at the center of the microchannel. The small and large particles relax towards the pressure nodes (dashed lines) at different rates and collection at different outlets achieves separation. and dipole scattering coefficient, respectively. Index 'p' denotes a particle property, while ' 0 ' denotes a liquid property. Symbols $\rho$ and $\kappa$ are used for density and compressibility, respectively. For our particles and frequency, the real part of the dipole scattering coefficient can be approximated by $f_{2}$ in Eq. (1), neglecting viscous effects. Previously we presented a different form of acoustic contrast factor in surface wave devices [15] as opposed to that widely used for bulk devices, hence the different scaling factor for the second term of the contrast factor in Eq. (1). Particles with positive contrast factor collect at the pressure nodes, while particles with negative contrast agglomerate at the pressure antinodes. The viscous, Stokes drag, force is given as:

$F_{\mathrm{drag}}=-6 \pi \eta a \dot{y}=-c_{\mathrm{visc}} \dot{y}$

where $\eta$ is the dynamic viscosity of the medium and $a$ is the particle radius and $\dot{y}$ is the time derivative of the position, i.e. the particle speed.

The primary acoustic radiation force in standing wave fields scales with the particle volume, or with the cube of the particle radius, while the viscous drag force is proportional to the particle size. Consequently, as the acoustic radiation force drags the particles with positive contrast towards the nodes, different particles experience different magnitude of acoustic and hydrodynamic forces and follow different trajectories. The same argument holds for density or compressibility, as the radiation force depends on these parameters, but not the viscous drag force. As a summary, separation of particles based on size, density or compressibility is possible.

The implementation of this time-of-flight (ToF) method can be seen in Fig 1. The particle mixture enters the device through the middle inlet, two sheath flows at the side inlets facilitate hydrodynamic focusing. The device width is chosen to be half of the wavelength, which is the separation distance of the nodes in the standing wave. The phase of the transducers is adjusted to have the antinode of the standing wave along the axis of the channel (dotted line in Fig. 1), while the two nodes are along the channel sides (dashed lines in Fig. 1). As a result of the force balance, the larger particles (in green) are displaced laterally to a larger distance compared to the smaller particles. The trifurcated outlet configuration allows the target particles to be collected at the two side outlets, while the waste exits through the middle outlet.

Inertial effects can be neglected for micrometer-size particles [18]. The mass times acceleration term in Newton's second law can therefore be dropped; the two forces balance one another and the trajectory of the particles is [17] 


$$
\begin{aligned}
y(t) & =\frac{1}{k_{y}} \tan ^{-1}\left[\tan \left(k_{y} y_{0}\right) \exp \left(2 k_{y} \frac{c_{\text {ac }}}{c_{\text {visc }}} t\right)\right] \\
& =\frac{1}{k_{y}} \tan ^{-1}\left[\tan \left(k_{y} y_{0}\right) \exp (\gamma t)\right] \\
\gamma & =2 k_{y} c_{\text {ac }} / c_{\text {visc }}
\end{aligned}
$$

where $y_{0}$ is the initial position of the particle at $t=0$. The $x$ axis of the coordinate system coincides with the antinode of the acoustic field at the axis of the channel. Equation (3) can be rearranged to obtain the settling time between an initial position $y_{0}$ and a final position $y$ :

$t=\frac{1}{\gamma} \ln \left[\frac{\tan \left(k_{y} y\right)}{\tan \left(k_{y} y_{0}\right)}\right]$.

As particles of different size or material have different $\gamma$ values, as shown in Eq. (3), they have different settling time even for the same initial and final positions, as illustrated by Eq. (4). We note here what has also been emphasized by Yang et al. [16]: neither the initial nor the final particle position can be at the nodes or antinodes if this time is to be evaluated. In these cases, the argument of the logarithm would be either zero or infinity. To avoid this issue, we take the settling time from the middle of the half inlet to the half of the outlet - none of these positions is a node or antinode.

Equation (3) can be used to obtain sensitivity information analytically of changing parameters when the partial derivatives are evaluated as detailed in Section 3.3.

\subsection{Phase modulated sorting}

In this section the phase modulated sorting method will be briefly summarized. A more in-depth overview is given in our previous works $[11,15]$.

The illustration of the phase modulated sorting can be seen in Fig. 2. In Fig. 2(a) one cycle of the phase pattern applied on one transducer is shown. During the ramping time, $t_{\text {ramp }}$, the phase is changed linearly from $0^{\circ}$ to $360^{\circ}$, and afterwards kept constant for $t_{\text {rest }}$ length. The pressure nodes within the microchannel move laterally as depicted in Fig. 2(b). At the start of the sorting process, all particles are located at the bottom pressure node at $y=-\lambda / 4$ (Fig. 2(c) left graph). Because of the interplay between acoustic and viscous forces, different particles are located on different sides of the pressure antinode (Fig. 2(c) middle graph) after the ramping cycle and are directed to different nodes during the resting cycle (Fig. 2(c) right graph) achieving sorting. In [15] we showed that phase modulation directly enters the argument of the acoustic radiation force

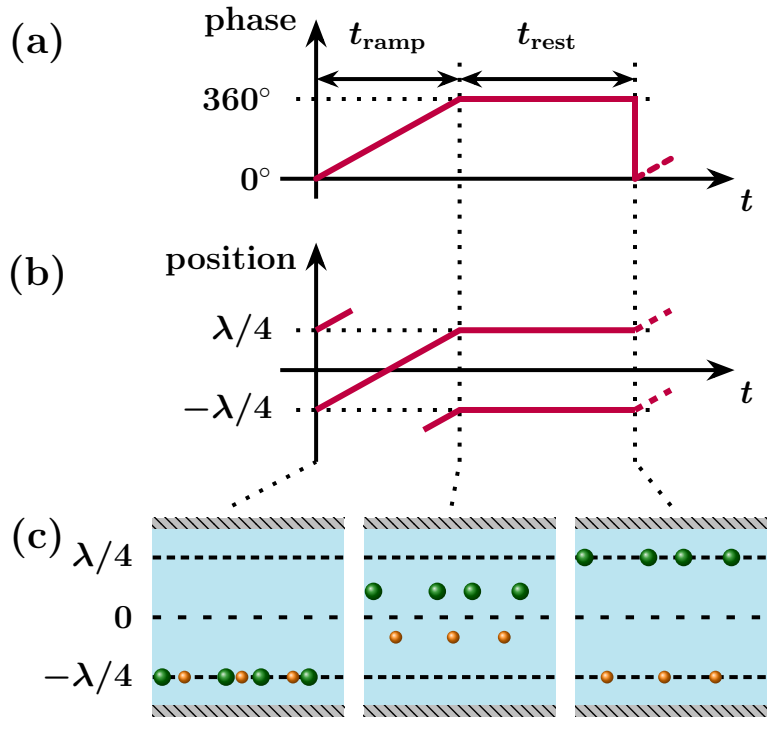

Fig. 2 The phase modulated sorting technique. (a) The phase pattern applied periodically on one transducer (b) The resulting movement of the nodes in the microchannel (c) Particle positions within the microchannel prior to sorting (left), after the ramping (middle) and after the resting time (right). Pressure nodes are indicated as densely dashed lines, while the pressure antinode is dotted. Particles assume different spatial locations after one full phase cycle, resulting in separation

$$
F_{\mathrm{ac}, y}(t)=c_{\mathrm{ac}} \sin \left(2 k_{y} y-s t\right)
$$

where $s$ is the phase modulation speed with $s \ll \omega$. Balancing the radiation force with the drag force by inertial approximation [18], the equation describing particle motion is directly obtained:

$$
y(t)=\frac{s\left(t-t_{\mathrm{s}}\right)}{2 k_{y}}+\frac{1}{k_{y}} \tan ^{-1}\left[\frac{\gamma-Z \tan \left(\frac{Z}{2}\left(c_{1}+t\right)\right)}{s}\right]
$$

where $Z=\sqrt{s^{2}-\gamma^{2}}$ and $t_{s}$ denotes the start of the phase shift. The constant $c_{1}$ is to satisfy initial conditions of particle position. For the above configuration, the particles start at the bottom node, $y=-\lambda / 4$, and $t_{\mathrm{s}}=0$ without the loss of generality (this would only imply a time shift). From $t=0$ and rearranging, the initial condition $c_{1}=\pi / Z$ is directly obtained.

The sorting in the phase modulated method is governed by the $\gamma$ parameter (similarly to the ToF case) and the ramping parameter $s$. The effect of various timing values on sorting was investigated before [19].

\section{Overview of sensitivity analysis}

For a function $f$ of several variables, $x_{1}, \ldots, x_{i}, \ldots, x_{n}$, the absolute sensitivity with respect to a single variable $x_{i}$ is defined as [20] 
$\Delta f(\boldsymbol{x})_{i}=\frac{\partial f(x)}{\partial x_{i}} \Delta x_{i}$

We can recast this equation into a form that contains the relative error of the function $f$ with respect to the variable $x_{i}$ :

$$
\frac{\Delta f(\boldsymbol{x})_{i}}{f(x)}=\frac{\partial f(x)}{\partial x_{i}} \frac{x_{i}}{f(x)} \frac{\Delta x_{i}}{x_{i}} .
$$

Throughout the theoretical analysis of stability, we investigate the system one variable at a time and use the above formula to obtain the relative error of particle trajectories given the partial derivative with respect to the variable in question and its relative error. We take this relative error of the final particle position as an estimate of the sensitivity.

To assess the quality of sorting, we used sorting efficiency and sorting purity, defined as ratios between particle counts collected at the various outlets [21]. The efficiency is the ratio of the target particles $(\mathrm{T})$ at the target outlet (@T) to the total number of target particles (at the target outlet, @T, and at the non-target outlet, @non-T); the purity is the ratio of the target particles at the target outlet to the total number of particles at the target outlet.

$$
\begin{array}{r}
\text { efficiency }=\frac{N_{\mathrm{T} @ \mathrm{~T}}}{N_{\mathrm{T} @ \mathrm{~T}}+N_{\mathrm{T} @ \text { non-T }}} . \\
\text { purity }=\frac{N_{\mathrm{T} @ \mathrm{~T}}}{N_{\mathrm{T} @ \mathrm{~T}}+N_{\text {non-T } @ \mathrm{~T}}} .
\end{array}
$$

Both purity and efficiency are high for a good sorting method.

\subsection{Extrinsic parameters}

The three extrinsic parameters investigated were the flow rate $(Q)$, acoustic energy density $\left(E_{\mathrm{ac}}\right)$ and focusing efficiency (or inlet width).

Increasing the flow rate results in particles exiting the device sooner and therefore being subjected to the acoustic field for a shorter time and being displaced by a smaller amount in the y-direction. Consequently, for the ToF method, we expect smaller number of target particles in the target region (reduced efficiency). Moreover, as for high purity sorting the relative number of target particles at the target outlet is higher than non-target particles, increasing flow rate will decrease the number of both target and non-target particles, resulting in an increase of purity. However, due to the periodic trapping of the PM method at the pressure nodes, and therefore stabilizing the position of the particles, we expect negligible dependence of any figure of merit on flow rate for the PM technique.
As the channel length has an equivalent effect on the sorting as changing the flow rate, we decided to exclude the investigation of the channel length.

The acoustic energy density has a similar effect to the flow rate: decreasing the energy density makes the particles travel less and consequently a drop in efficiency and increase in purity are observed. Due to the periodic forcing with the PM technique, again we expect little influence of the energy density. The acoustic radiation force has a direct dependence of energy density and quadratic dependence of pressure amplitude, and therefore we found it worthwhile to investigate dependence of this parameter.

Finally, the focusing efficiency is expected to have a similar effect for both sorting methods: increasing the inlet width would lower purity and efficiency since the particles have a more dispersed initial position and would follow trajectories with a larger spread.

\subsection{Intrinsic parameters}

The three intrinsic parameters investigated were the particle size, density and compressibility. During the manufacturing of microbeads, each of these vary randomly assuming nevertheless a Gaussian distribution, with well-defined mean and standard deviation [22].

As the ratio of the acoustic radiation and viscous drag forces is proportional to the square of the particle radius and proportional to the acoustic contrast factor, we expect a strong dependence on size and a moderate sensitivity to particle properties. As the principle of sorting is similar in the ToF and PM methods, no significant difference in sensitivity on intrinsic particle properties is anticipated.

\subsection{Analytical sensitivity of the time-of-flight method}

To be able to assess sensitivity with respect to various parameters, the partial derivative of Eq. (3) is required with respect to all variables of interest:

$$
\frac{\partial y(t)}{\partial t}=\frac{2 c_{\mathrm{ac}} \tan \left(k_{y} y_{0}\right) \exp (\gamma t)}{c_{\mathrm{visc}}\left[\tan ^{2}\left(k_{y} y_{0}\right) \exp ^{2}(\gamma t)+1\right]}
$$

and exchanging $t$ and $\gamma, c_{\mathrm{ac}}$ or $c_{\mathrm{visc}}$ gives

$$
\begin{aligned}
& \frac{\partial y(t)}{\partial \gamma}=\frac{t \tan \left(k_{y} y_{0}\right) \exp (\gamma t)}{k_{y}\left[\tan ^{2}\left(k_{y} y_{0}\right) \exp ^{2}(\gamma t)+1\right]} \\
& \frac{\partial y(t)}{\partial c_{\mathrm{ac}}}=\frac{2 t \tan \left(k_{y} y_{0}\right) \exp (\gamma t)}{c_{\mathrm{visc}}\left[\tan ^{2}\left(k_{y} y_{0}\right) \exp ^{2}(\gamma t)+1\right]} \\
& \frac{\partial y(t)}{\partial c_{\mathrm{visc}}}=-\frac{2 c_{\mathrm{ac}} t \tan \left(k_{y} y_{0}\right) \exp (\gamma t)}{c_{\mathrm{visc}}^{2}\left[\tan ^{2}\left(k_{y} y_{0}\right) \exp ^{2}(\gamma t)+1\right]} .
\end{aligned}
$$


Finally, the derivative with respect to the initial particle position:

$$
\frac{\partial y(t)}{\partial y_{0}}=\frac{\sec ^{2}\left(k_{y} y_{0}\right) \exp (\gamma t)}{\tan ^{2}\left(k_{y} y_{0}\right) \exp ^{2}(\gamma t)+1} \text {. }
$$

To obtain numerical sensitivity values and be able to compare the effect of the different parameters, we used the values that were also applied in the model as shown in Table 1. The parameters in Table 1 are either from the two reference papers provided in Section 4.1, or [23] as far as the material properties are concerned. First, based on the energy density, frequency and particle properties, we evaluated all variables for the larger $10 \mu \mathrm{m}$ sphere. We have $c_{\mathrm{ac}}=7.37 \mathrm{nN}$ for the acoustic force constant, $c_{\mathrm{visc}}=94.2 \mu \mathrm{N}$ for the viscous force constant, $k_{v}=20944 \mathrm{~m}^{-1}$ for the wavenumber on the surface. Using these, $\gamma=3.28$, and assuming that the particle travels from the middle of the half inlet $(0.0375 \lambda)$ to the middle of the outlet $(0.1875 \lambda)$, the settling time evaluates to $0.7 \mathrm{~s}$.

Firstly, we investigated the flow stability theoretically. Since the channel is more than twice wider than its height, and the flow speed is almost uniform towards the

Table 1 Parameters used in the numerical simulation. Values marked with a star $(*)$ are calculated based on other parameters and are presented to aid the reader

\begin{tabular}{|c|c|c|c|}
\hline Symbol & Description & $\begin{array}{l}\text { Value for } \\
\text { ToF sorter }\end{array}$ & $\begin{array}{l}\text { Value for } \\
\text { PM sorter }\end{array}$ \\
\hline$f$ & Frequency & \multicolumn{2}{|c|}{$13.3 \mathrm{MHz}$} \\
\hline$c_{\mathrm{s}}$ & $\begin{array}{l}\text { Speed of sound on the lithium } \\
\text { niobate surface }\end{array}$ & \multicolumn{2}{|c|}{$3990 \mathrm{~m} / \mathrm{s}$} \\
\hline \multirow[t]{7}{*}{$\lambda$} & Wavelength on surface & \multicolumn{2}{|c|}{$300 \mu \mathrm{m}$} \\
\hline & Width of channel & $\lambda / 2=150 \mu \mathrm{m}$ & $240 \mu \mathrm{m}$ \\
\hline & Shadow zone width & \multicolumn{2}{|c|}{$39 \mu \mathrm{m}$} \\
\hline & Height of channel & \multicolumn{2}{|c|}{$65 \mu \mathrm{m}$} \\
\hline & Length of active area & $1.62 \mathrm{~mm}$ & $3 \mathrm{~mm}$ \\
\hline & Inlet width & $22.5 \mu \mathrm{m}$ & $36 \mu \mathrm{m}$ \\
\hline & Inlet offset & $0 \mu \mathrm{m}$ & $-55 \mu \mathrm{m}$ \\
\hline$Q$ & Volumetric flow rate & \multicolumn{2}{|c|}{$0.4 \mu \mathrm{l} / \mathrm{min}=6.7 \mathrm{e}-12 \mathrm{~m}^{3} / \mathrm{s}$} \\
\hline$E_{\mathrm{ac}}$ & Acoustic energy density & \multicolumn{2}{|c|}{$1 \mathrm{~J} / \mathrm{m}^{3}$} \\
\hline$t_{\text {ramp }}$ & Ramping time & - & $1.7 \mathrm{~s}$ \\
\hline$t_{\text {rest }}$ & Rest time & - & $1 \mathrm{~s}$ \\
\hline$\rho_{0}$ & Density of water & \multicolumn{2}{|c|}{$998 \mathrm{~kg} / \mathrm{m}^{3}$} \\
\hline$\rho_{\mathrm{PS}}$ & Density of PS particle & \multicolumn{2}{|c|}{$1050 \mathrm{~kg} / \mathrm{m}^{3}$} \\
\hline$\kappa_{0}$ & Compressibility of water & \multicolumn{2}{|c|}{$457 \mathrm{TPa}^{-1}$} \\
\hline$\kappa_{\mathrm{PS}}$ & Compressibility of PS particle & \multicolumn{2}{|c|}{$172 \mathrm{TPa}^{-1}$} \\
\hline$N$ & $\begin{array}{l}\text { Number of simulations for } \\
\text { each case }\end{array}$ & \multicolumn{2}{|c|}{10000} \\
\hline$a_{i}$ & Particle radius & \multicolumn{2}{|c|}{3 and $5 \mu \mathrm{m}$} \\
\hline
\end{tabular}

centerline of the channel [11], we assumed the flow variance can be directly approximated by time variance. First evaluating Eq. (10) we obtain $\partial y / \partial t=55.5 \mu \mathrm{m} \cdot \mathrm{s}^{-1}$ and consequently $\Delta y / y=\partial y / \partial t \cdot \Delta t / t \cdot t / y=0.69 \Delta t / t$, or from our assumption $\Delta y / y=0.69 \Delta Q / Q$.

For stability against energy density, we can note that the acoustic force constant $\left(c_{\mathrm{ac}}\right)$ is directly proportional to the energy density. Therefore, stability against energy density is the same as against the acoustic force constant [20]. Using the same methodology as before, $\partial y / \partial c_{\mathrm{ac}}=5.28 \cdot 10^{3} \mathrm{~m} \cdot \mathrm{N}^{-1}$, and $\Delta y / y=0.69 \Delta c_{\mathrm{ac}} / c_{\mathrm{ac}}$, or $\Delta y / y=0.69 \Delta E_{0} / E_{0}$. This result is equivalent to the time (flow rate) dependence, which is expected, since $t$ and $c_{\text {ac }}$ appear equivalently in Eqs. (3), (10) and (11).

For the final extrinsic parameter, the inlet focusing stability, direct substitution into Eq. (12) yields $\partial y / \partial y_{0}=1.56$, and therefore $\Delta y / y=0.312 \Delta y_{0} / y_{0}$.

For size dependence a similar technique as for the energy density can be used [20]. As $\gamma$ is proportional to the square of the particle radius, $\partial \gamma / \partial a=2 \gamma / a$, and the size dependence can be obtained after calculating $\partial y / \partial \gamma=1.19 \cdot 10^{-5} \mathrm{~m} \cdot \mathrm{s}$, and substituting into $\Delta y / y=1.38 \Delta a / a$.

To approximate the density dependence, the derivative $\partial y / \partial \rho_{p}$ should be calculated. Here we can simplify utilizing the proportionality between $c_{\mathrm{ac}}$ and $\Phi_{\mathrm{ac}}$. Therefore, in the chain rule $\partial y / \partial \rho_{p}=\partial y / \partial c_{a c} \cdot \partial c_{a c} / \partial \Phi_{\mathrm{AC}} \cdot \partial \Phi_{\mathrm{AC}} / \partial \rho_{p}$ the second term can be substituted by $c_{\mathrm{ac}} / \Phi_{\mathrm{AC}}[20]$ and as $\partial y / \partial c_{a c}$ is known previously, only

$$
\begin{aligned}
\frac{\partial \Phi_{\mathrm{AC}}}{\partial \rho_{p}} & =-\frac{3}{2} \cos 2 \theta_{r} \frac{6 \rho_{0}}{\left(2 \rho_{p}+\rho_{0}\right)^{2}} \\
& \approx-6.74 \cdot 10^{-4} \mathrm{~kg}^{-1} \cdot \mathrm{m}^{3}
\end{aligned}
$$

needs to be derived. On substitution of the numerical values, we obtain $\partial y / \partial \rho_{p}=-4.46 \cdot 10^{-8} \mathrm{~m}^{4} \cdot \mathrm{kg}^{-1}$ and $\Delta y / y=-0.833 \Delta \rho_{p} / \rho_{p}$.

A similar methodology applied for the compressibility dependence gives

$$
\frac{\partial \Phi_{\mathrm{AC}}}{\partial \kappa_{p}}=-\frac{1}{\kappa_{0}} \approx 2.2 \cdot 10^{9} \mathrm{~Pa}
$$

and consequently, applying the chain rule, $\partial y / \partial \kappa_{p}=\partial y / \partial c_{\mathrm{ac}} \cdot c_{\mathrm{ac}} / \Phi_{\mathrm{AC}} \cdot \partial \Phi_{\mathrm{AC}} / \partial \kappa_{p}=1.46 \cdot 10^{5} \mathrm{~N} \cdot \mathrm{m}^{-1}$ and finally $\Delta y / y=0.45 \Delta \kappa_{p} / \kappa_{p}$.

The trajectories describing the PM method contain variables depending on several other parameters (such as $Z$ depending on $s$ and $\gamma$ ) which make calculation of the 
partial derivatives impractical. Moreover, due to the resting phase, approximation of sensitivity is difficult: a particle might get displaced more during the ramping phase, but stabilizes itself during resting. Therefore, we decided to omit analytical treatment of the PM method.

\section{Simulation setup}

In this section, we refer to the two articles used for the ToF and PM methods, detail the simulation parameters and comment on the anechoic corner that needs to be considered to accurately capture the surface wave behavior within the microchannel.

The simulation algorithm was a MATLAB implementation of the trajectory equations given by Eqs. (3) and (6) combined with fluid flow calculations as presented in [24], thus capturing the primary acoustic radiation force and viscous drag force. As a first step before each simulation, the random particle properties and initial time and position are generated. These are used in the analytical particle trajectory equations to obtain the $y(t)$ path of the individual particles. Next the generated particle trajectories are checked for entering the anechoic corner at the two sidewalls. If the particle $y$ position is closer to any of the walls than the width of the anechoic corner at time $t_{\mathrm{F}}$, then $y\left(t>t_{F}\right)=y\left(t_{F}\right)$, i.e. a freeze boundary condition is applied. Next, based on the volumetric flow rate and device geometry, the velocity field is calculated numerically up to the fifth order. Using this field and the $y(t)$ of the particles, the position along the device, $x(t)$, is obtained. Finally, the particles are counted at the two outlets.

This approach makes use of the analytical particle trajectories and the known radiation force on a spherical particle. A similar methodology can be used for non-spherical particles as well, the numerical calculation of radiation force in these cases was presented by Glynne-Jones et al. [25].

\subsection{Reference papers used, alignment of models}

We chose two works as references for the techniques where the device dimensions and operating conditions were similar enough that, with minimal modifications, a direct comparison was possible. The time-of-flight method was implemented using the work from Jo and Guldiken [14], while for the phase modulation we relied on a work by Simon et al. [11, 15]. Both references use 13.3 MHz operating frequency and a target outlet width that is half of the main channel width. There was a slight difference in channel height: 80 and $50 \mu \mathrm{m}$ for the time-of-flight and for the phase modulated method, respectively. Therefore, in both models we used the average $65 \mu \mathrm{m}$. The inlet focusing is hydrodynamic with the PM and acoustic with the ToF technique; we simply assumed that the sample inlet occupies $15 \%$ of the main channel width in both cases. Finally, the particles are considered to be perfectly suspended within the liquid and locate at the middle of the channel height.

For each simulation, 10,000 small $6 \mu \mathrm{m}$ and 10,000 large $10 \mu \mathrm{m}$ polystyrene particles were randomly dispersed within the inlet region. For the PM method, the particles entered the channel at a random time instant between 0 and $t_{\text {ramp }}$. The complete list of simulation parameters are shown in Table 1 for the two methods. The extrinsic parameters were simulated for $80 \%$ and $120 \%$ of their nominal values to obtain sensitivity for changes in both directions. For the intrinsic parameters, each of the 10,000 simulation steps used a physical property randomly selected following a Gaussian distribution with the nominal value as the mean and $20 \%$ of the nominal value as the standard deviation.

\subsection{The anechoic corner}

In a real surface acoustic wave microfluidics device, the channel walls shield the wave propagation, and the pressure field at the channel walls will mainly comprise one travelling wave component from the opposite direction [26]. This phenomenon is called an anechoic corner or shadow zone. As the illustration in Fig. 3 shows, in a region defined by the Rayleigh angle such as in Fig. 3(b), the pressure is much attenuated compared to the ideal standing wave pattern shown in Fig. 3(a), and the trapping is not perfect. Particles are pushed towards the channel walls due to acoustic streaming and the opposing travelling wave [27]. To take this effect into consideration, we calculated the maximum
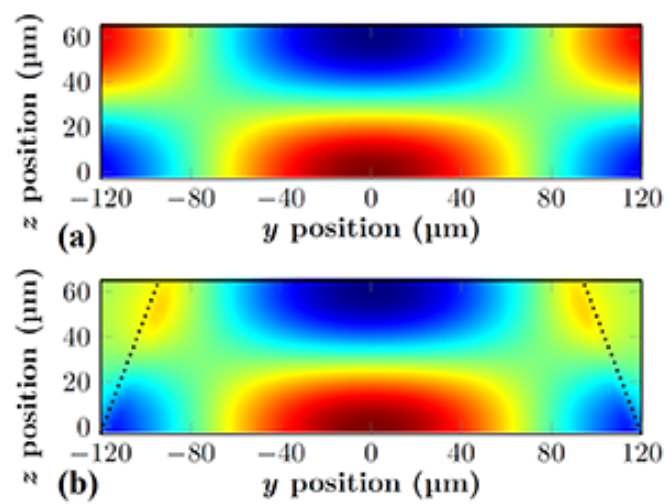

Fig. 3 Illustration of the anechoic corner or shadow zone. (a) Pressure distribution assuming perfect boundaries with no attenuation in the PDMS (b) pressure distribution due to attenuation in PDMS. Dashed lines indicate the approximate edges of the shadow zone 
width of the shadow zone, and with an additional $50 \%$ safety margin used it as a freeze boundary condition in the simulations. The width used in the simulations was $39 \mu \mathrm{m}$.

\section{Results}

\subsection{Time-of-flight method}

The simulation results for the time-of-flight method can be seen in Fig. 4. As a quick summary of the analytical predictions, that were derived in Section 3.3, the ratio of the relative error of the final particle position to the relative error of extrinsic parameters was estimated to have the following influence on sorting: 0.69, 0.69 and 0.312 for flow rate, energy density and inlet focusing, respectively. For example, for flow rate $\Delta y / y=0.69 \Delta Q / Q$. For the intrinsic parameters, the sensitivity values were $1.38,-0.833$ and 0.45 for size, density and compressibility, respectively.

The simulation results are generally in good agreement with the predictions and expectations of Section 3.3 when considering the strength of the individual parameters. Increasing flow rate (point $Q_{+20 \%}$ in Fig. 4), decreasing energy density $\left(\left(E_{\mathrm{ac}}\right)_{-20 \%}\right)$ or inlet focusing $\left(\left(y_{0}\right)_{-20 \%}\right)$ all decrease efficiency and increase purity. For higher flow rate and lower energy density, the particles are not allowed to move enough in the $y$-direction, which explains this change. However, for reduced inlet width we believe the decrease in efficiency is explained by the antinode position near the particle initial position: the particles move slower towards the sidewalls and consequently efficiency drops. Change of these parameters in the opposite direction increases efficiency and drops purity. As shown in Fig. 4, the change in purity is always more severe than the change in efficiency. Furthermore, the order of sensitivity of these parameters are in good alignment with the results of Section 3.3: highest sensitivity for flow rate and energy density, followed by a moderate sensitivity for inlet focusing.

Continuing with intrinsic parameters a more symmetric behavior can be observed. All data points ( $a, \rho$ and $\kappa)$ move towards the origin, and do not swing towards either the purity or efficiency side significantly. Again, good alignment with theoretical prediction can be seen: highest sensitivity is present for size-change, followed by density-change and a negligible compressibility-dependence.

\subsection{PM method}

Generally, the phase modulated method showed exceptional stability for most of the investigated parameters (Fig. 5). As predicted, the method is virtually insensitive to changes in extrinsic parameters such as flow rate, acoustic

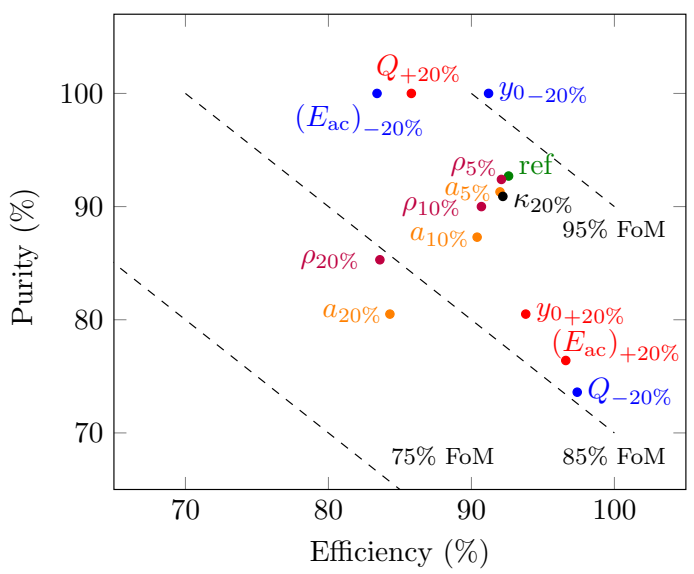

Fig. 4 Results of the sensitivity analysis for the time-of-flight method. Index: ref: reference simulation, Q: flow rate variation, $E_{\text {ac }}$ : acoustic energy variation, $y_{0}$ : inlet width variation. For these extrinsic simulations $-20 \%$ denotes simulation runs with $80 \%$ of the nominal value, $+20 \%$ denotes $120 \%$ of the nominal value. $a$ : size variance, $\rho$ : density variance, $\kappa$ : compressibility variance

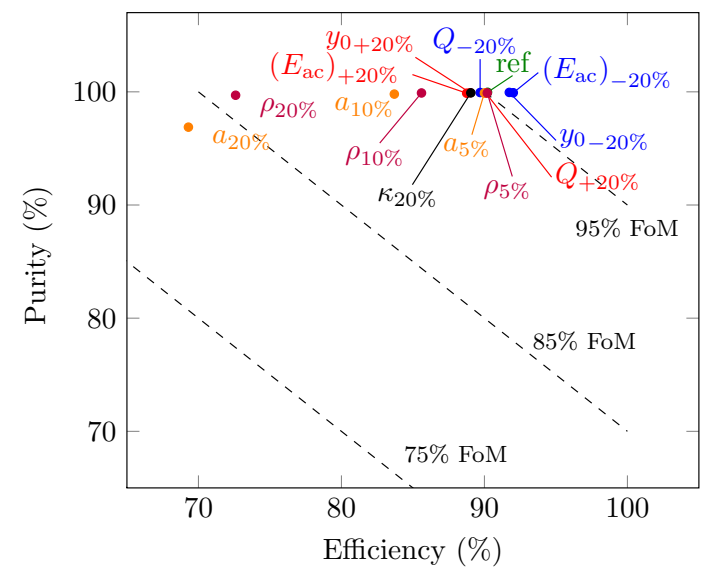

Fig. 5 Results of sensitivity analysis for the phase-modulated sorting. The notations are the same as in Fig. 4

energy density or inlet focusing, where the efficiency only changes by 3-4\%. However, the method was more prone to changes in particle size or density, in both cases the efficiency lowers to about $70 \%$. In these cases, the purity is still kept high, above $95 \%$. Gao et al. [28] argue that, in microfluidic sorting devices, a higher purity is more important, as it allows direct detection of the sorted entities at the outlet without the need of post-processing.

\section{Summary and conclusions}

In this paper, we presented the sensitivity analysis of two acoustic sorting methods in surface acoustic wave devices using numerical simulations. We also provided analytical prediction of the sensitivity of the time-of-flight method for various intrinsic and extrinsic parameters. 
The time-of-flight method showed higher sensitivity against extrinsic parameters, such as flow rate, acoustic energy density or inlet width, in these cases the change in efficiency or purity was $10-15 \%$. The dependence on intrinsic parameters such as size or density showed a more symmetric variation, lowering both purity and efficiency by $8-10 \%$.

For the phase-modulated sorting, minimal change in figures of merit was observed for the extrinsic parameters, lowering efficiency by only 3-4\%. However, the drop in efficiency could be as high as $25 \%$ for the variations against particle size or density. Even in these cases

\section{References}

[1] Gossett, D. R., Weaver, W. M., Mach, A. J., Hur, S. C., Tse, H. T., Lee, W., Amini, H., Di Carlo, D. "Label-free cell separation and sorting in microfluidic systems", Analytical \& Bioanalytical Chemistry, 397(8), pp. 3249-3267, 2010. https://doi.org/10.1007/s00216-010-3721-9

[2] Lenshof, A., Laurell, T. "Continuous separation of cells and particles in microfluidic systems", Chemical Society Reviews, 39(3), pp. 1203-1217, 2010.

https://doi.org/10.1039/b915999c

[3] Di Carlo, D. "Inertial microfluidics", Lab on a Chip, 9(21), pp. 3038-3046, 2009. https://oi.org/10.1039/b912547g

[4] Glynne-Jones, P., Hill, M. "Acoustofluidics 23: acoustic manipulation combined with other force fields", Lab on a Chip, 13(6), pp. 1003-1010, 2013. https://doi.org/10.1039/c31c41369a

[5] Wiklund, M. "Acoustofluidics 12: Biocompatibility and cell viability in microfluidic acoustic resonators", Lab on a Chip, 12(11), pp. 2018-2028, 2012. https://doi.org/10.1039/c2lc40201g

[6] Lenshof, A., Magnusson, C., Laurell, T. "Acoustofluidics 8: Applications of acoustophoresis in continuous flow microsystems", Lab on a Chip, 12(7), pp. 1210-1223, 2012 https://doi.org/10.1039/c2lc21256k

[7] Petersson, F., Nilsson, A., Holm, C., Jönsson, H., Laurell, T. "Continuous separation of lipid particles from erythrocytes by means of laminar flow and acoustic standing wave forces", Lab on a Chip, 5(1), pp. 20-22, 2005. https://doi.org/10.1039/b405748c

[8] Shi, J., Huang, H., Stratton, Z., Huang, Y., Huang, T. J. "Continuous particle separation in a microfluidic channel via standing surface acoustic waves (SSAW)", Lab on a Chip, 9(23), pp. 3354-3359, 2009. https://doi.org/10.1039/b915113c

[9] Destgeer, G., Lee, K. H., Jung, J. H., Alazzam, A., Sung, H. J. "Continuous separation of particles in a PDMS microfluidic channel via travelling surface acoustic waves (TSAW)", Lab on a Chip, 13(21), pp. 4210-4216, 2013. https://doi.org/10.1039/c3lc50451d the purity was above $95 \%$, and as Gao et al. [28] argue, high purity is often more beneficial than high efficiency. Therefore, the phase-modulated method can be a good solution due to its minimal sensitivity to extrinsic parameters and high purity operation for changes in intrinsic particle properties.

The results of this paper can be used to choose and design an appropriate sorting method for a given set of particles or cells with known variability to achieve either high purity or efficiency sorting. We would like to use the method in the future to automatically find the optimum device geometry for a given set of particles or cells.

[10] Nam, J., Lim, H., Kim, D., Shin, S. "Separation of platelets from whole blood using standing surface acoustic waves in a microchannel", Lab on a Chip, 11(19), pp. 3361-3364, 2011. https://doi.org/10.1039/c1lc20346k

[11] Simon, G., Pailhas, Y., Andrade, M. A. B., Reboud, J., MarquesHueso, J., Desmulliez, M. P. Y., Cooper, J. M., Riehle, M. O., Bernassau, A. L. "Particle separation in surface acoustic wave microfluidic devices using reprogrammable, pseudo-standing waves", Applied Physics Letters, 113(4), pp. 044101, 2018. https://doi.org/10.1063/1.5035261

[12] Ding, X. Y., Peng, Z. L., Lin, S. C. S., Geri, M., Li, S. X., Li, P., Chen, Y. C., Dao, M., Suresh, S., Huang, T. J. "Cell separation using tilted-angle standing surface acoustic waves", Proceedings of the National Academy of Sciences of the United States of America, 111(36), pp. 12992-12997, 2014. https://doi.org/10.1073/pnas.1413325111

[13] Li, P., Mao, Z., Peng, Z., Zhou, L., Chen, Y., Huang, P. H., Truica, C. I., Drabick, J. J., El-Deiry, W. S., Dao, M., Suresh, S., Huang, T. J. "Acoustic separation of circulating tumor cells", Proceedings of the National Academy of Sciences of the United States of America, 112(16), pp. 4970-4975, 2015. https://doi.org/10.1073/pnas.1504484112

[14] Jo, M. C., Guldiken, R. "Active density-based separation using standing surface acoustic waves", Sensors and Actuators A: Physical, 187, pp. 22-28, 2012. https://doi.org/10.1016/j.sna.2012.08.020

[15] Simon, G., Andrade, M. A. B., Reboud, J., Marques-Hueso, J., Desmulliez, M. P. Y., Cooper, J. M., Riehle, M. O., Bernassau, A. L. "Particle separation by phase modulated surface acoustic waves", Biomicrofluidics, 11(5), pp. 054115, 2017. https://doi.org/10.1063/1.5001998

[16] Yang, T., Vitali, V., Minzioni, P. "Acoustofluidic separation: impact of microfluidic system design and of sample properties", Microfluidics and Nanofluidics, 22(4), pp. 44, 2018. https://doi.org/10.1007/s10404-018-2063-3

[17] Bruus, H. "Acoustofluidics 7: The acoustic radiation force on small particles", Lab on a Chip, 12(6), pp. 1014-1021, 2012. https://doi.org/10.1039/c21c21068a 
[18] Bruus, H. "Acoustofluidics 10: Scaling laws in acoustophoresis", Lab on a Chip, 12(9), pp. 1578-1586, 2012. https://doi.org/10.1039/c2lc21261g

[19] Simon, G., Andrade, M. A. B., Roolvink, D., Cormack, P. A. G., Riehle, M. O., Bernassau, A. L. "A Deterministic Method for Particle Sorting by Dynamic Acoustic Fields", In: 2016 IEEE International Ultrasonics Symposium (IUS), Tours, France, 2016, pp. 1-4. https://doi.org/10.1109/ULTSYM.2016.7728638

[20] Fornasini, P. "The Uncertainty in Physical Measurements", Springer-Verlag New York, USA, 2008. https://doi.org/10.1007/978-0-387-78650-6

[21] Skotis, G. D., Cumming, D. R., Roberts, J. N., Riehle, M. O., Bernassau, A. L. "Dynamic acoustic field activated cell separation (DAFACS)", Lab on a Chip, 15(3), pp. 802-810, 2015. https://doi.org/10.1039/c4lc01153h

[22] Hipp, A. K., Storti, G., Morbidelli, M. "Acoustic Characterization of Concentrated Suspensions and Emulsions. 2. Experimental Validation", Langmuir, 18(2), pp. 405-412, 2002. https://doi.org/10.1021/la015541w

[23] Nama, N., Barnkob, R., Mao, Z., Kahler, C. J., Costanzo, F., Huang, T. J. "Numerical study of acoustophoretic motion of particles in a PDMS microchannel driven by surface acoustic waves", Lab on a Chip, 15(12), pp. 2700-2709, 2015. https://doi.org/10.1039/c5lc00231a
[24] Bruus, H. "Theoretical Microfluidics", Oxford University Press, Oxford, UK, 2008

[25] Glynne-Jones, P., Mishra, P. P., Boltryk, R. J., Hill, M. "Efficient finite element modeling of radiation forces on elastic particles of arbitrary size and geometry", The Journal of the Acoustical Society of America, 133(4), pp. 1885-1893, 2013. https://doi.org/10.1121/1.4794393

[26] Devendran, C., Albrecht, T., Brenker, J., Alan, T., Neild, A. "The importance of travelling wave components in standing surface acoustic wave (SSAW) systems", Lab on a Chip, 16(19), pp. $3756-$ 3766, 2016.

https://doi.org/10.1039/c6lc00798h

[27] Destgeer, G., Ha, B. H., Park, J., Jung, J. H., Alazzam, A., Sung, H. J. "Microchannel Anechoic Corner for Size-Selective Separation and Medium Exchange via Traveling Surface Acoustic Waves", Analytical Chemistry, 87(9), pp. 4627-4632, 2015. https://doi.org/10.1021/acs.analchem.5b00525

[28] Gao, Y., Li, W., Pappas, D. "Recent advances in microfluidic cell separations", Analyst, 138(17), pp. 4714-4721, 2013. https://doi.org/10.1039/c3an00315a 